\title{
Unintended Childbearing and Knowledge of Emergency Contraception in a Population-Based Survey of Postpartum Women
}

\author{
Kimberley A. Goldsmith • Laurin J. Kasehagen • \\ Kenneth D. Rosenberg · Alfredo P. Sandoval •
}

Jodi A. Lapidus

Published online: 23 October 2007

(C) Springer Science+Business Media, LLC 2007

\section{Erratum to: Matern Child Health J \\ DOI 10.1007/s10995-007-0252-x}

In this article, line 116 should have read as follows: $\leq 30 \%$.

The online version of the original article can be found under doi: 10.1007/s10995-007-0252-x.

K. A. Goldsmith · K. D. Rosenberg · J. A. Lapidus Department of Public Health and Preventive Medicine,

Oregon Health \& Sciences University, Portland, OR, USA

\section{Present Address:}

K. A. Goldsmith

Medical Research Council Biostatistics Unit, Cambridge, UK

L. J. Kasehagen · K. D. Rosenberg $(\bowtie) \cdot$ A. P. Sandoval Office of Family Health, Oregon Department of Human Services, 800 NE Oregon Street, Suite 850, Portland, OR 97232, USA

e-mail: ken.d.rosenberg@state.or.us 